\title{
Efectos de la Tutoría entre Iguales como Estrategia de Educación Inclusiva en el Alumnado con Trastorno del Espectro Autista de Educación Infantil y Primaria
}

\author{
The Effects of Peer Tutoring as an Inclusive Educational \\ Strategy in Students with Autism Spectrum Disorder of Pre- \\ School and Elementary School
}

\author{
Elena Martínez * \\ David Duran
}

Universitat Autònoma de Barcelona, España

\begin{abstract}
Este artículo presenta una revisión de estudios que ponen en práctica la tutoría entre iguales, una estrategia educativa que consiste en la creación de parejas en las que un alumno desarrolla el rol de tutor y el otro el de tutorado, y donde ambos comparten un objetivo común de aprendizaje. Aunque esta práctica educativa es recomendada como una metodología para la inclusión, sus efectos en alumnado con Trastorno del Espectro Autista (TEA) están todavía poco estudiados. Por este motivo, el objetivo de la presente revisión bibliográfica es estudiar cuáles son los efectos y beneficios concretos de esta metodología en alumnado con TEA de Educación Infantil y Primaria. En la primera búsqueda, se localizaron 132 artículos, de los cuales 10 cumplían con los criterios de inclusión. Los resultados muestran como la tutoría entre iguales puede ser una práctica efectiva para la mejora de las habilidades sociales y académicas del ámbito de las matemáticas, educación física y lengua de los estudiantes con TEA. También se refleja como los compañeros del alumnado con TEA viven la experiencia de tutoría de forma positiva.
\end{abstract}

Descriptores: Tutoría entre iguales; Trastorno del espectro autista; Habilidades sociales; Habilidades académicas; Educación inclusiva.

This article presents a review of studies that put in practice peer tutoring. Peer tutoring is a learning methodology based on the creation of pairs where one student adopts the role of tutor and the other the role of tutee, and where both students share a common learning objective. Although this educational practice is an inclusive methodology, its effects with students who have Autism Spectrum Disorder (ASD) are scarcely investigated. Due to this, the purpose of this systematic review is to study which are the effects and benefits of using this educational practice with students with ASD. At the beginning, 132 articles were located and 10 of them were included in the revision as matched with the inclusion criteria. The main results of the research show that peer tutoring can be an effective and inclusive strategy to increase social skills and academic skills, related to Physical Education, Mathematics and Language, of students with ASD. Moreover, it is also noted that the peers of students with ASD live the peer tutoring experience in a very positive way.

Keywords: Peer tutoring; Autism spectrum disorder; Social skills; Academic skills; Inclusive education.

*Contacto: elena.maar8@gmail.com

Recibido: 


\section{Introducción}

La educación inclusiva hace referencia al proceso de mejora de la enseñanza con el objetivo de ofrecer una respuesta educativa de calidad a todo el alumnado, independientemente de sus características (Giné, 2009). A pesar de que existen diversas metodologías que promueven la inclusión, en este artículo nos centraremos específicamente en una de ellas: la tutoría entre iguales, una práctica ampliamente recomendada por expertos en educación, como la European Agency for Development in Special Needs Education (2001). Según dicha organización, diversos estudios demuestran como la tutoría entre iguales es efectiva en las áreas cognitivas y socioemocionales del proceso de aprendizaje de los alumnos.

El objetivo de este artículo es realizar una revisión bibliográfica de estudios que pongan en práctica la tutoría entre iguales con alumnado con necesidades educativas especiales derivadas del Trastorno del Espectro Autista (TEA). Con esta revisión se pretende analizar cuáles son los efectos de esta estrategia en el alumnado con TEA.

\subsection{La tutoría entre iguales como respuesta educativa inclusiva en las aulas}

Los inicios de la tutoría entre iguales se sitúan en la Revolución Francesa, donde la falta de maestros y el incremento del alumnado propició el hecho de que los alumnos más ventajosos asumieran el rol de profesores de los otros alumnos, sobre los cuales se tenía expectativa de aprendizaje, y no sobre el alumno tutor. El concepto de tutoría entre iguales concebido hoy en día se aleja de esta unidireccionalidad del aprendizaje, dado que se ha demostrado que el proceso de ayuda que un alumno ofrece a otro puede generar oportunidades de aprendizaje para él y que enseñar puede ser una buena manera de aprender (Duran, 2017).

Desde un enfoque centrado en la educación formal, la tutoría entre iguales se define como un método de aprendizaje entre iguales basado en la creación de parejas con una relación asimétrica (procedente de los roles respectivos de tutor y tutorado), que comparten un objetivo común que se alcanza a partir de un marco de relación planificado por el profesor (Topping et al., 2015). La relación uno a uno de la tutoría entre iguales posibilita una alta participación y una comunicación más directa que permite altos niveles de modelaje y demostración personalizada. El tutorado aprende en tanto que recibe ayuda personalizada y ajustada de su tutor, mientras que éste lo hace gracias a las posibilidades de aprender enseñando (Duran, 2015).

Las prácticas de tutoría entre iguales son muy ricas y se pueden clasificar en función de diversos criterios. Dado que el objetivo de esta revisión se centra en el ámbito escolar, cabe destacar dos criterios que parecen especialmente importantes en este contexto (Duran, 2015):

- Edad o curso: parejas de distinta edad o curso (cross-age tutoring) o de edades y cursos similares (same-age tutoring). Los trabajos de investigación sobre ambas tipologías indican que la calidad del aprendizaje no depende tanto de la diferencia de edad entre el alumnado, sino de garantizar "distancia" entre las habilidades del tutor y el tutorado.

- Continuidad de rol: rol permanente (no hay intercambio de roles entre tutor y tutorado) o rol recíproco (intercambio periódico de los roles de tutor y tutorado). 
Las prácticas cross-age tutoring promueven el rol permanente, mientras que las same-age tutoring, pueden adoptar tanto roles permanentes como recíprocos.

Una vez analizado el concepto de tutoría entre iguales, es necesario conocer las evidencias que la apoyan como una metodología para la inclusión. Disponemos de revisiones de investigaciones y metaanálisis que apuntan en ese sentido. La revisión de estudios de Greenwood y otros (1990), centrada tanto en contextos de escuelas ordinarias como de centros de educación especial, concluye que la tutoría entre iguales es tanto o más efectiva que las prácticas tradicionales mediadas por el profesor. El metaanálisis de BowmanPerrot y otros (2013) concluye que los beneficios de la tutoría son perceptibles también para los alumnos con discapacidad, así como para aquellos con desórdenes emocionales y conductuales. Además, señala que las mejoras académicas de estos estudiantes se deben al apoyo adicional que reciben de sus compañeros. Finalmente, el metaanálisis de Leung (2015) concluye que la tutoría se muestra académicamente más efectiva en estudios en los cuales la mitad o menos de los alumnos pertenecen a grupos con vulnerabilidad, frente a otros en los que la mayoría de los estudiantes son catalogados de esta forma. Por lo tanto, se trata de una metodología que saca provecho pedagógico de la diversidad.

\subsection{El alumnado con trastorno del espectro autista}

Según la American Psychiatric Association (2013), el TEA se caracteriza por la presencia de dos síntomas nucleares: déficits persistentes en la comunicación y en la interacción social, en varios contextos, que no se explica por un retraso general del desarrollo; y existencia de patrones restrictivos y repetitivo de conducta o intereses muy fijados.

Como es sabido, el concepto de espectro hace referencia a la heterogeneidad del trastorno, en tanto que la forma y el grado de los déficits señalados se pueden desarrollar de formas muy diversas. Las características interindividuales de las personas con TEA varían en función de la intensidad, el nivel de inteligencia y la presencia de síntomas secundarios o asociados. Entre estos últimos destacan el trastorno por déficit de atención e hiperactividad, el trastorno obsesivo compulsivo, trastornos alimentarios, así como elevados niveles de ansiedad (Romero et al., 2016). En el caso de los síntomas propios del trastorno, los déficits en la interacción social pueden ser muy variables. En algunos casos se puede presentar poco o nulo interés en establecer relaciones sociales, mientras que en otros se puede manifestar deseo en la interacción social que se ve dificultado por el déficit en la comprensión de normas sociales. También se puede observar ausencia de juego simbólico e imitativo social, propio del nivel de desarrollo infantil. En cuanto a las alteraciones en la comunicación, puede producirse desde una ausencia total del habla verbal, hasta un buen desarrollo del lenguaje oral, pero con alteraciones en la interacción recíproca (López et al., 2009).

En las últimas décadas, la detección del trastorno ha crecido notablemente, afectando este a un $1 \%$ de la población aproximadamente (Wong et al., 2014). Al trasladar este dato a los centros educativos, se observa que el número de alumnos con este trastorno también ha incrementado y, por lo tanto, es necesario buscar prácticas en la escuela ordinaria que promuevan su participación y aprendizaje. Las Peer-Mediated Instruction and Intervention $(P M I I)$ son un ejemplo de intervención inclusiva para el alumnado con TEA y se encuentran entre la lista de Evidence-Based Practices de la National Professional Development Center on Autism Spectrum Disorders (Wong et al., 2014). Las PMII se definen como un conjunto de estrategias educativas basadas en la interacción entre compañeros, con el 
objetivo de promover las habilidades sociales, adquirir nuevos comportamientos y aprender nuevas habilidades académicas. Existen diversas tipologías de PMII:

- Social Skills Training Groups: creación de pequeños grupos de alumnos con el objetivo de practicar una determinada habilidad social.

- Peer Modeling Interventions: creación de pequeños grupos en los cuales uno o más alumnos actúan como modelos de comportamiento apropiado. Los compañeros con TEA los observan de forma directa (Vivo Peer Modeling), o bien a través de un vídeo (Vídeo Peer Modeling), con el objetivo de imitarlos.

- Peer Tutoring Interventions: creación de parejas en las cuales un miembro asume el rol de tutor de un compañero. Este rol de tutor puede ser asumido tanto por el alumno sin necesidades educativas especiales, como por el alumno con TEA.

- Classwide Peer Tutoring Interventions: práctica en la cual todo un grupo clase participa en la tutoría entre iguales.

La efectividad de las PMII en alumnado con TEA ha sido corroborada a través de diferentes revisiones de estudios. En la revisión de Wong y otros (2014) identifican las PMII como una de las Evidence-Based Practices seleccionadas por docentes a la hora de diseñar programas de intervención destinados a alumnos con TEA de 3 a 18 años. Los autores afirman que las PMII pueden ser utilizadas para desarrollar habilidades sociales, comunicativas, de juego y de lectura, así como habilidades académicas. El metaanálisis Bene y otros (2014) concluye que las PMII son efectivas a la hora de mejorar las habilidades comunicativas, académicas y sociales del alumnado con TEA de entre 5 y 12 años.

Watkins y otros (2015) realizan una revisión de estudios y sostienen que las PMII son prácticas prometedoras a la hora de augmentar las interacciones sociales del alumnado con TEA. En la misma línea, la revisión de Chang y Locke (2016) concluye que las PMII pueden mejorar las habilidades sociales de niños y adolescentes en el contexto escolar. Según los autores, los compañeros de aula son un recurso humano muy potente y accesible que puede ofrecer al alumnado con TEA un buen modelo de comportamiento social, así como posibilidades de poner en práctica las habilidades sociales que estos vayan adquiriendo. Una revisión más reciente (Aldabas, 2020) señala que las PMII pueden mejorar el comportamiento social y las habilidades comunicativas del alumnado con TEA.

\subsection{Propósito de la investigación}

Así pues, la efectividad de las PMII en alumnado con TEA está ampliamente estudiada de forma general. No obstante, ya se ha comentado que las PMII no describen un solo tipo de intervención, sino que engloban diferentes metodologías, siendo una de ellas la tutoría entre iguales. El motivo de esta revisión recae en la existencia de pocos estudios que se centren específicamente en estudiar la tutoría entre iguales y sus efectos con el alumnado con TEA. Por lo tanto, el objetivo de este artículo es realizar una revisión bibliográfica de estudios que pongan en práctica específicamente la tutoría entre iguales, con el fin de conocer cuáles son los efectos y beneficios que esta provoca en el alumnado con TEA de Educación Infantil y Primaria. A través de esta revisión se intentará dar respuesta a las siguientes cuestiones:

- ¿Qué tipologías de tutoría entre iguales y roles (tutor/tutorado) se dan entre el alumnado, con y sin TEA, participante en los diferentes estudios? 
- ¿Cómo incide la tutoría entre iguales en las habilidades sociales del alumnado con TEA?

- ¿En qué grado la tutoría entre iguales mejora las habilidades académicas del alumnado con TEA?

- ¿Qué visión sobre la experiencia de tutoría entre iguales tienen los compañeros del alumnado con TEA que participan en este tipo de prácticas?

\section{Método}

\section{Diseño}

Se ha realizado una revisión bibliográfica sistematizada de artículos publicados en Peerreviewed journals que proporcionen datos sobre los efectos de la tutoría entre iguales en el alumnado con TEA de Educación Infantil y Primaria. Las bases de datos utilizadas han sido PsycINFO (base de datos de la American Psychological Association), ERIC (Education Resources Information Center) y Web of Science (Thomson Reuters). En todas ellas, la búsqueda se ha limitado a estudios publicados entre los años 1985 y 2020.

\section{Estrategia de búsqueda}

En las bases de datos PsycINFO y ERIC, la búsqueda se ha limitado al tipo de publicación "Peer Reviewed Journal' y tipo de documento "Journal Article". Además, la búsqueda también se ha acotado al período escolar "Preschool: 3-5 years" y "School: 6-12 years". En ambas bases de datos se han utilizado los dos siguientes bloques de palabras clave, en lengua inglesa:

- Palabras clave sobre tutoría entre iguales: "peer tutoring" OR "peer teaching" OR "peer-mediated instruction" OR "peer-mediated intervention".

- Palabras clave sobre TEA: "autism" OR "asd" OR "autism spectrum disorder".

En la búsqueda, las palabras clave se han combinado de la siguiente forma: (Bloque A) AND (Bloque B). En la base de datos Web of Science, las palabras utilizadas para localizar los artículos han sido "peer tutoring" AND "autism".

\section{Criterios de inclusión y exclusión}

El proceso de selección de artículos se ha realizado de acuerdo con los criterios de inclusión y exclusión que se presentan a continuación. En la búsqueda se han incluido:

- Artículos que presentan una intervención en un contexto escolar de Educación Infantil o Primaria, con alumnado participante de entre 3 y 12 años.

- Artículos que presentan una intervención educativa basada en la tutoría entre iguales, entendiendo esta como un método de aprendizaje basado en la creación de parejas, donde un alumno es el tutor y el otro es el tutorado y ambos comparten un objetivo de aprendizaje común.

- Artículos que presentan una muestra en la cual al menos un alumno está diagnosticado con TEA.

- Artículos de investigación empírica que proporcionan datos sobre el efecto de la tutoría entre iguales en los alumnos, y especialmente en el alumnado con TEA. 
Por otro lado, los criterios de exclusión establecidos son los siguientes:

- Artículos que, a pesar de llevar a cabo prácticas de tutoría en el aula, su objetivo se focaliza en estudiar otras variables no relacionadas con los efectos de esta práctica.

- Artículos que en su muestra incluyen alumnos participantes con otras necesidades educativas especiales que no son el TEA.

- Artículos que son revisiones bibliográficas

Selección de artículos

Una vez realizada la búsqueda, se localizaron 132 artículos (62 PsycINFO, 39 ERIC y 31 Web of Science), después de eliminar aquellos que estaban duplicados en las diferentes bases de datos. En la primera fase de selección de artículos, después de revisar títulos y abstracts, han sido excluidos 114 estudios que no cumplían criterios de inclusión. Seguidamente, se ha realizado un segundo cribaje del primer grupo de 18 artículos, a partir de la lectura completa de todos ellos, con el fin de realizar una selección más rigurosa. Finalmente, 10 artículos que cumplían con criterios de inclusión han pasado a formar parte del banco de documentos de la revisión bibliográfica (6 PsycINFO, 2 ERIC y 2 Web of Science).

\section{Resultados}

A continuación, de forma previa al análisis de los resultados, se presentan sintéticamente cada uno de los 10 artículos seleccionados. Con el fin de observar una síntesis más detallada de las características de los estudios vea el Anexo I.

El primer artículo de la revisión (Ayvazo y Ward, 2006) analiza los efectos de la tutoría entre iguales en el área de Educación Física, como una estrategia inclusiva para alumnado con TEA. Los participantes son cuatro alumnos de 8 años, dos de los cuales están diagnosticados de TEA. El diseño de intervención se basa en una línea base inicial, seguida de dos intervenciones de tutoría entre iguales.

Gillies (2013) analiza cuáles son los efectos de la cross-age tutoring en el desarrollo del comportamiento del alumnado con TEA. La muestra se compone de seis alumnos, tres de ellos con edades de entre 4 y 5 años y diagnosticados con autismo; y los tres restantes de 9 años. El diseño experimental es similar al anterior e incluye además una prueba de mantenimiento final.

El tercer artículo incluido en la revisión (Jones, 2007) valora el impacto de la tutoría entre iguales en aquellos alumnos que hacen de tutores de sus compañeros con TEA. Para ello, participan 12 alumnos diagnosticados con TEA de edades entre 4 y 10 años y 27 alumnos de edades entre 10 y 11 años, los cuales adoptan el rol de tutores. Para la recopilación de datos, se utiliza un cuestionario que recoge las experiencias de los tutores.

Kamps y otros (1994) se centran en valorar los efectos de la tutoría entre iguales en las habilidades lectoras y sociales de tres estudiantes con autismo y sus compañeros. Los participantes son, por un lado, tres alumnos con TEA de 8 y 9 años y 14 compañeros de aula de estos. La metodología consiste en una línea base inicial, seguida de la intervención de tutoría basada en actividades de lengua. Después se llevan a cabo actividades de tiempo libre, en las cuales se valoran las habilidades sociales. 
Kamps y otros (1999) investigan el uso de la tutoría entre iguales como método para promover la interacción entre estudiantes con TEA y sus compañeros sin necesidades educativas especiales. Entre los participantes encontramos dos alumnos diagnosticados con TEA, de 9 años, y 11 alumnos sin necesidades educativas especiales, de 6 y 9 años. El proceso metodológico es similar al anterior estudio, pero con dos líneas base y dos intervenciones de tutoría intercaladas.

El estudio de Kamps y otros (1989) analiza la efectividad de la tutoría entre iguales en la adquisición de habilidades académicas y sociales de alumnado con TEA. La muestra se compone de dos alumnos con TEA de 9 y 11 años, y dos alumnos sin necesidades educativas especiales, de 10 años. La metodología cuenta con una línea base, el entrenamiento de los tutores y una posterior intervención de tutoría.

Laushey y Helfin (2000) tienen como propósito determinar si la tutoría entre iguales incrementa la generalización de habilidades sociales entre el alumnado. Participaron dos alumnos con TEA de 5 años y otros compañeros sin necesidades educativas especiales de entre 5 y 6 años. En la práctica, se realizan dos líneas base y dos intervenciones de tutoría, de forma intercalada.

McGee y otros (1992) analizan la eficacia de la tutoría entre iguales a la hora de promover interacciones recíprocas entre alumnado con TEA. Los participantes son tres alumnos diagnosticados con TEA de 3 , 4 y 5 años, y 5 alumnos sin necesidades educativas especiales de entre 3 y 4 años. En el proceso metodológico, además la línea base y la intervención de tutoría, se llevan a cabo dos fases de extinción finales en las cuales el profesor deja de guiar a los alumnos progresivamente.

Petursdottir y otros (2007) implementan la tutoría entre iguales en actividades de lectura con el fin de analizar los efectos en las interacciones sociales de alumnos con TEA y sus compañeros. En estas actividades participan un alumno con TEA de 5 años y tres compañeros de 5 años. A diferencia de los estudios anteriores, los autores introducen el Programmed common stimuli (piezas de juego de construcción) en las sesiones de tutoría para analizar su impacto.

El último estudio de la revisión (Whitaker, 2004) tiene como objetivo incrementar, a través de la tutoría, la frecuencia en la que el alumnado con TEA inicia la comunicación y la atención conjunta. Los participantes son 10 alumnos diagnosticados con TEA, de entre 6 y 7 años, y 10 alumnos sin necesidades educativas especiales de 11 años. Este estudio no lleva a cabo línea base, sino que directamente se centra en el entrenamiento de los tutores y en la posterior intervención basada en la tutoría.

A continuación, se trata de responder con los resultados a las cuatro preguntas de investigación.

\section{1. ¿Qué tipologías de tutoría entre iguales y roles (tutor/tutorado) se dan entre el alumnado, con y sin TEA, participante en los diferentes estudios?}

Para responder a esta pregunta se han tenido en cuenta las tipologías de tutoría entre iguales que hacen referencia a la edad de los participantes y a la continuidad de roles.

Los resultados muestran que cuatro estudios utilizan la tipología de tutoría entre iguales same-age y fija (Ayvazo y Ward, 2006; Kamps et al., 1989; Laushey y Helfin, 2000; McGee et al., 1992). El rol fijo que adopta el alumnado en estos estudios es también coincidente; los participantes tutorados siempre son el alumnado con TEA, mientras que el rol de tutor 
es adoptado por otros compañeros del grupo clase que no presentan ninguna necesidad educativa especial. Dos estudios más (Kamps et al., 1994; Petursdottir et al., 2007) también hacen uso de la tutoría entre iguales same-age, pero recíproca. En estos casos, el rol de todo el alumnado participante, con y sin TEA, se va intercambiando periódicamente. Por lo tanto, el alumnado con TEA adopta tanto el rol de tutor como de tutorado. Los estudios restantes (Gillies, 2013; Jones, 2007; Kamps et al., 1999; Whitaker, 2004) hacen uso de la tutoría entre iguales cross-age y fija.

Como coincidencia entre las investigaciones de Gillies (2013), Jones (2007) y Whitaker (2004), el rol de tutor es siempre llevado a cabo por el alumno de mayor edad que no presenta TEA y el rol de tutorado lo adoptan los participantes con TEA de menor edad. Como excepción de este patrón, en el estudio de Kamps y otros (1999), el alumnado con TEA de mayor edad es quien adopta el rol de tutor, mientras que los tutorados son los estudiantes de menor edad que no presentan ninguna necesidad educativa especial. Según los autores, que el alumnado con TEA haya adoptado el rol de tutor ha generado beneficios muy positivos. El hecho de ser tutores les ha permitido a ayudar a otros compañeros, en lugar de recibir ellos la ayuda y, por lo tanto, les ha hecho sentirse capaces de colaborar en la mejora del aprendizaje de sus tutorados. Asimismo, Kamps y otros (1999) destacan la capacidad del alumnado con TEA a la hora de tutorizar a otro compañero de menor edad, ya que en todos los casos la tutoría se ha mostrado efectiva con dichos roles.

A partir de este análisis de tipología de tutoría entre iguales, se observa un bajo porcentaje de estudios (30\%) en los cuales el alumno con TEA adopta el rol de tutor (Kamps et al., 1994, 1999; Petursdottir et al., 2007). Por otro lado, el patrón de roles que predomina en el resto de las investigaciones (70\%) es que el alumno con TEA sea el tutorado y el alumno sin TEA el tutor.

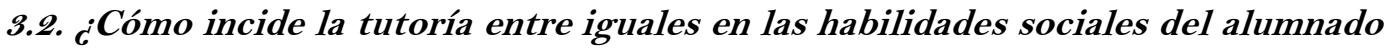 con TEA?}

Se han identificado siete estudios (Gillies, 2013; Kamps, 1994, 1999; Laushey y Helfin, 2000; McGee et al., 1992; Petursdottir et al., 2007; Whitaker, 2004) que proporcionan datos sobre la incidencia de la tutoría entre iguales en las habilidades sociales del alumnado con TEA. Como coincidencia entre los diferentes estudios, los resultados apuntan a que la calidad y la cantidad de interacciones sociales del alumnado con TEA mejoran, en mayor o menor grado, cuando se produce la intervención de tutoría entre iguales, en comparación con una línea base. Veámoslo con algo de detalle.

Gillies (2013) señala que la tutoría entre iguales provoca un notable cambio en el comportamiento social de los participantes con TEA, específicamente cambios en su autonomía y en la relación con sus iguales. Durante la línea base, en el contexto de la biblioteca, los participantes con TEA manifiestan actitudes poco ajustadas y realizan escasos comentarios verbales. Durante la intervención de tutoría, los alumnos establecen mayor comunicación verbal y contacto ocular con los tutores, y permanecen más tiempo al lado de estos de forma voluntaria, escuchándolos y siguiendo sus indicaciones con interés. También se observa como los alumnos con TEA manifiestan predisposición para compartir los libros de la biblioteca con sus tutores y, según el autor, se sienten calmados, seguros e interesados durante la experiencia de tutoría.

En la misma línea, Kamps y otros (1994) concluyen que establecer sesiones de tutoría entre iguales provoca que en las sesiones posteriores a la tutoría, basadas en juego libre no estructurado, se provoquen interacciones sociales más duraderas entre los alumnos. En 
la primera línea base de sesiones de tiempo libre no precedidas de tutoría, la duración de las interacciones sociales para los tres alumnos con TEA es de 50", 40" y 25" respectivamente, durante 5 minutos de observación. En la primera intervención de tutoría, basada en una sesión de juego posterior a una sesión de tutoría, la duración de las interacciones aumenta para los tres participantes, con 144", 120" y 145", respectivamente. En la segunda línea base, la media de tiempo de interacción social disminuye, mientras que vuelve a aumentar de nuevo en la segunda intervención de tutoría. Según los autores, el aumento de las interacciones sociales en los alumnos con TEA que hemos observado, se debe a la oportunidad de interacción que se les proporciona a través de la tutoría entre iguales. Así pues, el uso de esta metodología es un punto de partida realmente importante para el alumnado con TEA, teniendo en cuenta que este presenta dificultades en las habilidades sociales.

Los resultados de Kamps y otros (1994) difieren con la investigación de Petursdottir y otros (2007). Este equipo concluye que las sesiones de tutoría entre iguales, basadas en $K$ Pals Reading Activities (actividades basadas en la identificación de fonemas, lectura de palabras clave y lectura de frases) no provocan que en las sesiones de juego libre posteriores a la tutoría haya un incremento de las interacciones sociales. Dichas diferencias entre ambos estudios, según Petursdottir y otros (2007), pueden deberse a dos motivos. En primer lugar, las actividades de tutoría de Kamps y otros (1994) eran menos guiadas que las sesiones de tutorías basadas en K-Pals y, por lo tanto, propiciaban posiblemente más oportunidades de interacción social. Por otro lado, Kamps y otros (1994) tenían en consideración las interacciones no verbales a la hora de cualificar un comportamiento como interacción social, mientras que Petursdottir y otros (2007) solo recogían las declaraciones verbales.

Aun así, los resultados de Petursdottir y otros (2007) señalan que introducir estímulos relacionados con el juego en las sesiones de tutoría sí que puede aumentar las interacciones sociales entre el alumnado con TEA y sus compañeros. En las sesiones de juego libre, seguidas de tutoría entre iguales con estímulos de juego, se observa como el tiempo de interacción social aumenta para los participantes. En cambio, cuando se retiran los estímulos de juego durante la tutoría, en las sesiones de juego posteriores disminuyen la cantidad de interacciones sociales. No obstante, los autores remarcan que introducir estímulos de juego durante la actividad lectora de tutoría provoca que el alumno permanezca más tiempo jugando que leyendo.

Kamps y otros (1999) realizan una investigación siguiendo un patrón metodológico parecido a Kamps y otros (1994). En la línea base se mide el nivel de interacción social en sesiones de juego libre anteriores a la cross-age tutoring, mientras que en la intervención se mide la interacción social en sesiones de juego libre posteriores a la cross-age tutoring. En la primera línea base, la media de tiempo de interacción para las dos participantes con TEA es de 31 ” y 95”, durante 5 minutos de observación. En la primera intervención, el tiempo de interacción aumenta a 164" y 154", datos que vuelven a disminuir en la segunda línea base y vuelven a aumentar en la segunda intervención: 139" y 148”. Los resultados nos indican como la tutoría aumenta la media de tiempo de interacción en sesiones de tiempo libre que preceden a la tutoría. Esto se debe a la potencialidad que tiene combinar actividades académicas y sociales, ya que los compañeros del aula son buenos modelos de cooperación y de adquisición de habilidades sociales para el alumnado con TEA. 
Laushey y Helfin (2000) muestran que, con la intervención de tutoría, el alumnado participante con TEA lleva a cabo un mayor porcentaje de comportamientos sociales. Dichos comportamientos hacen referencia a cuatro habilidades sociales pertenecientes al nivel de Educación Infantil: pedir un objeto y responder correctamente a la demanda; obtener la atención de un igual; esperar el turno de conversación y mirar en la dirección de la persona que habla. Para los dos alumnos con TEA, el porcentaje de comportamientos sociales en la línea base (29\% y $28 \%$ ) se dobla en la intervención de tutoría entre iguales ( $75 \%$ y $66 \%)$. Mientras que durante la línea base los alumnos participan en menos turnos de palabra, establecen poco contacto ocular y tienen baja tolerancia a la espera, en la intervención de tutoría se observan mayores indicios de las habilidades sociales definidas anteriormente. En palabras de los autores, estos positivos resultados pueden deberse a las oportunidades que nos da la tutoría entre iguales de entrenar al alumnado, con y sin TEA, y de estructurar las situaciones de interacción. Además, el hecho de que la tutoría se realice con todo el grupo clase favorece que el alumnado con TEA observe un modelo más representativo de la variabilidad de comportamientos sociales que se dan en esta, ayudándolo, por tanto, a desarrollar interacciones sociales más naturales.

McGee y otros (2012) muestra como las interacciones recíprocas de los alumnos con TEA con sus compañeros de clase incrementan durante las sesiones de tutoría. Dichas interacciones consisten en la demanda verbal por parte del alumno con TEA o dirigida al alumno con TEA, y la respuesta positiva a la demanda. El porcentaje de interacciones recíprocas positivas de los tres estudiantes con TEA aumentan durante la intervención de tutoría (25\%, 35\% y 13\%), en comparación con la línea base (1\%, $7 \%$ y 0\%). Además, en la Fading phase 1 y 2 , donde la actividad del docente disminuye, las interacciones durante la tutoría continúan en niveles elevados, por encima de la línea base (Fading phase 1: 31\%, $25 \%$ y $21 \%$; Fading phase 2: 25\%, 41\% y 9\%). Por lo tanto, el hecho de generar situaciones, como la tutoría, en las cuales se enseñe al tutor como debe actuar y comunicarse con su tutorado favorece el incremento de las iniciaciones y respuestas verbales por parte del alumnado.

Por último, Whitaker (2004) expone que la tutoría entre iguales incide en las habilidades sociales del alumnado con TEA, concretamente relacionadas con el juego compartido, la comunicación y la atención conjunta. En datos recogidos sobre juego compartido, se muestra como durante las sesiones de tutoría hay signos de participación, motivación y compromiso por parte del alumnado con TEA. Por otro lado, el contacto ocular y la sonrisa social son poco frecuentes durante todo el proyecto. En cuanto a la comunicación, la frecuencia con la que los alumnos con TEA hacen demandas a sus tutores se triplican en las sesiones de tutoría. También se produce un aumento de comentarios espontáneos. En relación con la atención, durante la tutoría no se produce ningún cambio respecto a los niveles de atención conjunta del alumnado con TEA. Así pues, los autores exponen que gracias la preparación de los alumnos y el modelo que imitación que nos proporciona la tutoría, los alumnos sin necesidades educativas especiales son capaces de promover ciertos comportamientos sociales en extensos períodos de juego compartido.

\section{3. ¿En qué grado la tutoría entre iguales mejora las habilidades académicas del alumnado con TEA?}

Las investigaciones de Ayvazo y Ward (2006), Kamps y otros (1994) y Kamps y otros (1989) muestran como la práctica de tutoría ha provocado mejora en ciertas habilidades académicas del alumnado con TEA participante en los estudios. En palabras de algunos 
de los autores, el hecho de llevar a cabo una actividad académica que incluya una interacción entre los alumnos altamente estructurada, como es el caso de la tutoría, da lugar a que se produzcan los beneficios académicos que expondremos a continuación. Veamos los resultados de los estudios uno a uno.

Ayvazo y Ward (2006) exponen que la tutoría entre iguales puede beneficiar a un mayor desarrollo de las habilidades académicas del alumnado con TEA en el ámbito de Educación Física. Durante la primera intervención de tutoría, el número total de capturas de pelota aumenta para los dos participantes con TEA, un 25\% y 50\% respectivamente, en comparación con la línea base. En la segunda intervención, mientras que en el primer alumno no hay un incremento el número total de capturas, ni de capturas correctas, el otro sí que vuelve a aumentar los dos parámetros. Según los autores, si el nivel de participación es un indicador de inclusión, se puede concluir que las sesiones de gran grupo son una estrategia inclusiva para los participantes. En cambio, aunque los resultados son variables, se ha observado cómo hacer uso de la tutoría entre iguales puede comportar mejoras en las habilidades deportivas.

Kamps y otros (1994) concluye que la tutoría entre iguales es una estrategia que puede mejorar las habilidades académicas relacionadas con el ámbito lingüístico, del alumnado con TEA. Con el fin de valorar los efectos de la tutoría, los autores se centran en tres variables: número de palabras leídas correctamente por minuto, número de errores por minuto y número de respuestas correctas en la comprensión lectora. Durante las intervenciones de tutoría, el número de palabras leídas aumenta para los tres estudiantes con TEA. Los errores por minuto disminuyen solo en algunos participantes durante la tutoría. En cuanto a la comprensión lectora, la tutoría provoca un aumento del porcentaje de respuestas correctas. Por lo tanto, se concluye que la práctica de tutoría puede comportar mejoras en la precisión y fluidez lectora, así como en la comprensión lectora. No obstante, los resultados que relacionan la tutoría y el número de errores son variables.

En la línea del estudio anterior, Kamps y otros (1989) indican que estudiantes con TEA pueden aprender habilidades académicas lingüísticas y matemáticas con la ayuda de sus compañeros tutores sin necesidades educativas especiales. Los estudiantes participantes realizan las siguientes actividades: Money skills, Language qüestions o Language opposites y Oral reading/comprehension. En la primera actividad, Money skills, la tutoría mejora notablemente las habilidades matemáticas de los alumnos, ya que durante esta las respuestas correctas aumentan un 50\%, en comparación con la línea base. En la segunda actividad, los resultados mejoran de nuevo con la intervención de tutoría. En la actividad Oral reading/comprehension, la tutoría provoca un aumento del número de palabras leídas correctamente, una disminución de los errores por minuto y un incremento de las respuestas correctas en la comprensión lectora. En conclusión, los autores exponen que la tutoría entre iguales es una práctica que promueve la creación de espacios de aprendizaje inclusivos en una escuela ordinaria.

\section{4. ¿Qué visión sobre la experiencia de tutoría entre iguales tienen los compañeros del alumnado con TEA que participan en este tipo de prácticas?}

Tres artículos de la revisión bibliográfica (Gillies, 2013; Jones, 2007; Whitaker, 2004) reflejan como los compañeros del alumnado con TEA, participantes en los estudios, viven la experiencia de tutoría entre iguales de forma muy positiva. 
Gillies (2013) expone que la cross-age tutoring genera notables efectos positivos, tanto en los tutores sin necesidades educativas especiales, como en los tutorados con TEA. En las experiencias recogidas en un diario de los estudiantes, los tutores exponen que se sienten importantes y confiados realizando su rol; también se sienten conectados y comprometidos con sus tutorados. Por otro lado, se reporta también la buena percepción que manifiestan los tutores en relación con los tutorados; estos se centran en describir los puntos fuertes de los compañeros con TEA y en aquello que les gusta, sin mencionar en ninguna ocasión los puntos débiles.

Jones (2007) muestra otro ejemplo de cómo los tutores de alumnos con TEA tienen una visión positiva de la tutoría. En las encuestas realizadas a los tutores, estos exponen que han disfrutado de la experiencia de ser tutores y les ha gustado el hecho de poder jugar con los compañeros con TEA, ayudarlos y animarlos a hacer actividades. El 89\% de los tutores expone que adoptar el rol de tutor les ha ayudado en diferentes aspectos, como por ejemplo a entender que todas las personas somos diferentes; a entender mejor el TEA; a ser más pacientes; a sentirse bien con ellos mismos y a ser más responsables. Según los autores, una limitación de la investigación ha sido el uso de cuestionarios en vez de entrevistas personales, ya que estas últimas podrían haber proporcionado información más detallada.

Whitaker (2004) expone que, durante las prácticas de tutoría entre iguales, todos los alumnos tutores sin TEA demostraron un alto nivel de compromiso y participación con sus tareas. Además, todos los tutores, a excepción de uno, reportaron que la tutoría fue una experiencia desafiante pero altamente satisfactoria.

\section{Conclusiones}

Los resultados de la revisión bibliográfica apoyan la idea de que la tutoría entre iguales puede ser una práctica educativa capaz de mejorar ciertas habilidades sociales y académicas del alumnado con TEA de Educación Infantil y Primaria. Ciertamente, se ha constatado como la calidad y la cantidad de interacciones sociales del alumnado con TEA mejoran cuando se produce la intervención de tutoría entre iguales, a causa de las oportunidades de interacción que esta metodología proporciona. Este hecho se refleja tanto en un mayor número de comportamientos sociales, así como en un incremento en el tiempo de interacción social. En el caso de las habilidades académicas, se han observado mejoras en las áreas de Educación Física, Matemáticas y Lengua. Estos resultados, teniendo en cuenta que la tutoría entre iguales es una tipología de $P M I I$, concuerdan con estudios anteriores que afirman que las PMII son intervenciones eficaces a la hora de mejorar las habilidades académicas y sociales del alumnado con TEA (Bene et al., 2014; Chang y Locke, 2016; Watkins et al., 2015).

En relación con el rol del alumnado, las pocas investigaciones en las cuales el alumno con TEA hace de tutor demuestran beneficios muy potentes, tanto para él mismo, como para sus compañeros. Al realizar el rol de tutor, el alumno con TEA siente que es capaz de proporcionar ayuda, mientras que el alumno sin necesidades educativas especiales aprende a valorar la diversidad de forma positiva. Esta idea concuerda con Roscoe y Chi (2007), los cuales exponen que cuando un alumno con necesidades educativas especiales adopta el rol de tutor, los resultados de la práctica pueden ser positivos. El hecho de ejercer el rol de tutor permite al alumno explicar y cuestionar a su tutorado, lo cual implica que 
previamente ha tenido que construir e integrar conocimientos. Además, durante el proceso de tutorizar se activa el pensamiento metacognitivo del alumno.

Como limitaciones de la revisión, dado que esta se ha realizado únicamente con diez estudios, hay que ser prudentes a la hora de generalizar los resultados obtenidos. Es cierto que las investigaciones nos han aportado evidencias de mejoras académicas y sociales, pero hay que tener en cuenta que estas se han realizado con muestras reducidas de alumnos, y con metodologías y técnicas de recogida de datos muy diversas, que pueden influir en los resultados. A pesar de los prometedores resultados, aún quedan muchas puertas para investigar los efectos de la tutoría en el alumnado con TEA, teniendo en cuenta otras habilidades académicas y sociales y con diseños de investigación más rigurosos, que permitan a la vez, combinados con metodologías cuantitativas, explicar el porqué de dichas mejoras.

Otra de las limitaciones de la revisión es la falta de resultados relacionados con las habilidades académicas del alumnado sin necesidades educativas especiales, compañeros del alumnado con TEA. Si bien en algunas investigaciones se proporcionaban datos sobre este aspecto, esta información no se ha tenido en cuenta en la revisión, dado que no era objetivo del trabajo. Por este motivo, sería recomendable que futuras investigaciones se centrasen en estudiar también los efectos académicos y sociales de este alumnado. Constatar que los alumnos tutores pueden aprender enseñando a sus compañeros tutorados, incluso cuando estos tienes necesidades especiales, puede ser un elemento potente a favor de la presencia y participación de alumnado con más necesidad de ayuda, y por lo tanto una línea de avance hacia la inclusión (Duran, 2014).

Aun así, los resultados disponibles para el alumnado con TEA indican que la tutoría puede ser una buena práctica educativa para avanzar en la inclusión del alumnado, coincidiendo con las revisiones generales de Greenwood y otros (1990), Bowman-Perrot y otros (2013) y Leung (2015). Si llevar a cabo prácticas de tutoría entre iguales en el aula ordinaria permite generar mayores oportunidades de interacción entre los estudiantes y, consecuentemente, promover mejoras en el ámbito académico y social de alumnado con TEA, su implementación puede significar un paso más en el avance hacia su inclusión.

\section{Referencias}

Aldabas, R. (2020). Effectiveness of peer-mediated interventions (PMIs) on children with autism spectrum disorder (ASD): A systematic review. Early Child Development and Care, 190(10), 1586-1603. https://doi.org/10.1080/03004430.2019.1580275

American Psychiatric Association. (2013). Diagnostic and statistical manual of mental disorders. American Psychiatric. https://doi.org/10.1176/appi.books.9780890425596

Ayvazo, S. y Ward, P. (2006). Classwide peer tutoring in physical education: Assessing its effects with kindergartners with autism. Adapted Physical Activity Quarterly, 23, 233-244. https://doi.org/10.1123/apaq.23.3.233

Bene, K., Banda, D. R. y Brown, D. (2014). A meta-analysis of peer-mediated instructional arrangements and autism. Review Journal of Autism and Developmental Disorders, 1(2), 135142. https://doi.org/10.1007/s40489-014-0014-9

Bowman-Perrot, L., Davis, H., Vannest, K., Williams, L., Greenwood, C. y Parker, R. (2013). Academic benefits of peer tutoring: A meta-analytic review of single-case research. $S$ chool Psychology Review, 42, 39-55. https://doi.org/10.1080/02796015.2013.12087490 
Chang, Y. C. y Locke, J. (2016). A systematic review of peer-mediated interventions for children with autism spectrum disorder. Research in Autism Spectrum Disorders, 27, 1-10. https://doi.org/10.1016/j.rasd.2016.03.010

Duran, D. (2014). Aprenseñar: Evidencias e implicaciones educativas de aprender enseñando. Narcea.

Duran, D. (2015). Tutoría entre iguales: Compartir la capacidad de enseñar con los alumnos. En R. Mayordomo y J. Onrubia (Coords.), El aprendizaje cooperativo (pp. 191-228). Editorial UOC.

Duran, D. (2017). Learning-by-teaching: Evidence and implications as a pedagogical mechanism. Innovations in Education and Teaching International, 54(5), 476-484. https://doi.org/10.1080/14703297.2016.1156011

European Agency for Development in Special Needs Education. (2001). Inclusive education and effective classroom practices. C.J.W. Meijer.

Gillies, A. (2013). The cross-age tutoring experience for students with and without disabilities. International Research in Early Childhood Education, 4(1), 105-126.

Giné, C. (2009). Aportaciones al concepto de inclusión. La posición de los organismos internacionales. En C. Giné, D. Duran, J. Font y E. Miquel (Coords.), La educación inclusiva: De la exclusión a la plena participación de todo el alumnado (13-24). Horsori.

Greenwood, C. R., Carta, J. J. y Kamps, D. (1990). Teacher-mediated vs. peer-mediated instruction: A review of educational advantages and disadvantages. En H. C. Foot, M. J. Morgan y R. H. Shute (Eds.), Children helping children (pp. 177-205). John Wiley \& Sons.

Jones, V. (2007). I felt like I did something good. The impact on mainstream pupils of a peer tutoring programme for children with autism. British Journal of Special Education, 34(1), 39. https://doi.org/10.1111/j.1467-8578.2007.00447.x

Kamps, D. M., Barbetta, P. M., Leonard, B. R. y Delquadri, J. (1994). Classwide peer tutoring: An integration strategy to improve reading skills and promote peer interactions among students with autism and general education peers. Journal of Applied Behavior Analysis, 27(1), 49-61. https://doi.org/10.1901/jaba.1994.27-49

Kamps, D. M., Dugan, E., Potucek, J. y Collins, A. (1999). Effects of cross-age peer tutoring networks among students with autism and general education students. Journal of Behavioral Education, 9(2), 97-115. https://doi.org/10.1023/A:1022836900290

Kamps, D., Locke, P., Delquadri, J. y Hall, R. V. (1989). Increasing academic skills of students with autism using fifth grade peers as tutors. Education and Treatment of Children, 12(1), 38-51.

Laushey, K. M. y Heflin, L. J. (2000). Enhancing social skills of kindergarten children with autism through the training of multiple peers as tutors. Journal of Autism and Developmental Disorders, 30(3), 183-193. https://doi.org/10.1023/A:1005558101038

Leung, K. C. (2015). Preliminary empirical model of crucial determinants of best practice for peer tutoring on academic achievement. Journal of Educational Psychology, 107, 558-579. https://doi.org/10.1037/a0037698

Longwill, A.W. y Kleinert, H. (1998). The unexpected benefits of high school peer tutoring. Teaching Exceptional Children, 30(4), 60-65. https://doi.org/10.1177/004005999803000413

López, S., Rivas, R., y Taboada, E. (2009). Revisiones sobre el autismo. Revista Latinoamericana de Psicología, 41(3), 555-570.

McGee, G. G., Almeida, M. C., Sulzer-Azaroff, B. y Feldman, R. S. (1992). Promoting reciprocal interactions via peer incidental teaching. Journal of Applied Behavior Analysis, 25(1), 117-126. https://doi.org/10.1901/jaba.1992.25-117 
Petursdottir, A. L., McComas, J., McMaster, K. y Horner, K. (2007). The effects of scripted peer tutoring and programming common stimuli on social interactions of a student with autism spectrum disorder. Journal of Applied Behavior Analysis, 4O(2), 353-357.

https://doi.org/10.1901/jaba.2007.160-05

Romero, M., Aguilar, J. M., Del-Rey-Mejías, A., Mayoral, F., Rapado, M., Peciña, M. y Lara, J. P. (2016). Psychiatric comorbidities in autism spectrum disorder: A comparative study between DSM-IV-TR and DSM-5 diagnosis. International Journal of Clinical and Health Psychology, 16(3), 266-275. https://doi.org/10.1016/j.ijchp.2016.03.001

Roscoe, R. y Chi, M. (2007). Understanding tutor learning: Knowledge-building and knowledge telling in peer tutors' explanations and questions. Review of Educational Research, 77(4), 534574. https://doi.org/10.3102/0034654307309920

Topping, K., Duran, D. y Van Keer, H. (2015). Using peer tutoring to improve reading skills. Routledge. https://doi.org/10.4324/9781315731032

Watkins, L., O’Reilly, M., Kuhn, M., Gevarter, C., Lancioni, G. E., Sigafoos, J. y Lang, R. (2015). A review of peer-mediated social interaction interventions for students with autism in inclusive settings. Journal of autism and developmental disorders, 45(4), 1070-1083. https://doi.org/10.1007/s 10803-014-2264-x

Whitaker, P. (2004). Fostering communication and shared play between mainstream peers and children with autism: Approaches, outcomes and experiences. British Journal of Special Education, 31(4), 215-222. https://doi.org/10.1111/j.0952-3383.2004.00357.x

Wong, C., Odom, S. L., Hume, K. Cox, A. W., Fettig, A., Kucharczyk, S. y Schultz, T. R. (2014). Evidence-based practices for children, youth, and young adults with autism spectrum disorder. The Frank Porter Graham Child Development Institute.

https://doi.org/10.1007/s 10803-014-2351-z

\section{Breve CV de los/as autores/as}

\section{Elena Martínez Arroyo}

Maestra de Educación Primaria, Especialidad pedagogía terapéutica. Grado en Educación Primaria en la Universitat Autònoma de Barcelona, Mención en Necesidades Educativas Específicas. Participó junto a David Duran en el Congreso Internacional sobre participación social y educación inclusiva: Encuentros sobre equidad, diversidad y educación inclusiva: Aprendiendo de las experiencias de alumnado con TEA y de sus familias, en la mesa de comunicaciones "Efectos de la tutoría entre iguales como estrategia de educación inclusiva en alumnado con trastorno del espectro autista de educación infantil y primaria”. Email: elena.maar8@gmail.com

ORCID ID: https://orcid.org/OOOO-0002-4541-1247

\section{David Duran Gisbert}

Doctor en Psicología. Profesor del Departamento de Psicología Básica, Evolutiva y de la Educación de la Universitat Autónoma de Barcelona. Ha trabajado como profesor de enseñanza secundaria $\mathrm{y}$ ha realizado formación de profesorado en el ámbito de la enseñanza inclusiva y del aprendizaje cooperativo. Es coordinador del Grupo de Investigación sobre Aprendizaje entre Iguales UAB. Investigador principal del Proyecto "Observación entre iguales como desarrollo profesional docente: hacia una cultura colaborativa” (PID2020-113719RB-IOO). Docente del Máster Interuniversitario de 
Psicología de la Educación y coordinador de la asignatura Aprendizaje Cooperativo y Tutoría entre Iguales. Cuenta con muchas publicaciones sobre aprendizaje entre iguales y educación inclusiva, temáticas en las que ha desarrollado actividades de formación del profesorado. Email: david.duran@uab.cat

ORCID ID: https://orcid.org/0000-0002-0640-3834 


\section{Anexo I}

\section{Cuadro 1}

Principales características de los estudios de la revisión bibliográfica

\begin{tabular}{|c|c|c|c|}
\hline Reference & Purpose of the research & Participants & Intervention design / procedures \\
\hline $\begin{array}{l}\text { 1. Ayvazo } \\
\text { and Ward } \\
(2006)\end{array}$ & $\begin{array}{l}\text { To assess the effects of Classwide Peer } \\
\text { Tutoring as an inclusion strategy for } \\
\text { children with autism in physical education. }\end{array}$ & $\begin{array}{l}2 \text { children diagnosed } \\
\text { with ASD: } \\
\text { - Ben, 8-year-old. } \\
\text { - Peter, 8-year-old. } \\
2 \text { typically } \\
\text { developing children: } \\
\text { - Sarah, 8-year-old. } \\
\text { - Holly, 8-year-old. }\end{array}$ & $\begin{array}{l}\text { Experimental Design: A-B-A-C } \\
\text { A. Baseline: Whole Group Direct Instruction (WGDI). } \\
\text { - Training of tutors: tutors received 30-min training prior to the beginning of } \\
\text { CWPT. } \\
\text { B. Intervention 1: Classwide Peer Tutoring } 1 \text { (CWPT 1): The peer tutor asked } \\
\text { his or her partner to perform the task and waited a short time for a response ( } 4 \\
\text { seconds). If no response occurred, the tutor assisted the partner to perform the } \\
\text { task. } \\
\text { C. Intervention 2: Classwide Peer Tutoring } 2 \text { (CWPT 2): The tutors focused on } \\
\text { their own performance with the goal of modelling both the behaviour and the } \\
\text { on-task engagement. }\end{array}$ \\
\hline $\begin{array}{l}\text { 2. Gillies } \\
(2013)\end{array}$ & $\begin{array}{l}\text { To assess which effect a cross-age tutoring } \\
\text { intervention have on the progress of } \\
\text { students' with autism Individualized } \\
\text { Education Plan goals related to behaviour. }\end{array}$ & $\begin{array}{l}3 \text { children with ASD: } \\
\text {-Michael, 5-year-old. } \\
\text { - Mollie, 4-year-old. } \\
\text {-Jason, 5-year-old. } \\
3 \text { typically } \\
\text { developing children: } \\
\text {-Julie, Shawn and } \\
\text { Janie, 9-year-old. }\end{array}$ & $\begin{array}{l}\text { Multiple baseline design: A-B-C } \\
\text { A. Baseline: The target behaviour for the three tutees was to follow the direction } \\
\text { to get a book from the bookshelf, without the tutors. } \\
\text { - Pretraining: Before cross-age tutoring, the researcher trained the tutors in the } \\
\text { strategy they had to use when working with their tutees. } \\
\text { B. Cross-age tutoring intervention: The cross-age tutors use the least-to-most } \\
\text { prompting strategy with their tutees in the library. } \\
\text { C. Maintenance probe: it was collected at one month post the final intervention } \\
\text { session. }\end{array}$ \\
\hline $\begin{array}{l}\text { 3. Jones } \\
(2007)\end{array}$ & $\begin{array}{l}\text { To consider the impact of peer tutoring } \\
\text { children with autism on the peer tutors. }\end{array}$ & $\begin{array}{l}12 \text { children } \\
\text { diagnosed with } \\
\text { ASD, aged } 4 \text { to } 10 \\
\text { years old. } \\
27 \text { typically } \\
\text { developing children, }\end{array}$ & $\begin{array}{l}\text { There were implemented a wide variety of sessions and activities where children } \\
\text { from Year } 6 \text { class were peer tutors. These peer tutors had a basic understanding } \\
\text { of autism but were not specifically trained in how to play with children with } \\
\text { autism. } \\
\text { There was and adult who supported the interactions of the children with ASD } \\
\text { through the peer tutors. }\end{array}$ \\
\hline
\end{tabular}




\begin{tabular}{|c|c|c|c|}
\hline Reference & Purpose of the research & Participants & Intervention design / procedures \\
\hline & & $\begin{array}{l}\text { aged } 10 \text { or } 11 \text { years } \\
\text { old. }\end{array}$ & $\begin{array}{l}\text { The children were offered different choices of activities within sessions, such as } \\
\text { building activities, car races, ball games, dressing up... } \\
\text { Finally, the Year } 6 \text { peer tutors were asked to complete a questionnaire that } \\
\text { explored their experiences and feelings about being a peer tutor. }\end{array}$ \\
\hline $\begin{array}{l}\text { 4. Kamps et } \\
\text { al. (1994) }\end{array}$ & $\begin{array}{l}\text { To measure the effects of a Class Wide Peer } \\
\text { Tutoring program on the reading skills and } \\
\text { social interactions of } 3 \text { students with autism } \\
\text { and their classroom peers. }\end{array}$ & $\begin{array}{l}3 \text { children diagnosed } \\
\text { with ASD: } \\
\text { - Mike, 8-year-old. } \\
\text { - Adam, 8-year-old. } \\
\text { - Pete, a 9-year-old. } \\
\text { 14 typically } \\
\text { developing } \\
\text { students. }\end{array}$ & $\begin{array}{l}\text { Multiple baseline design: A-B } \\
\text { A. Baseline: Reading instruction consisted of teacher-directed lessons using a } \\
\text { basal reading series with individual variation in activities among the three } \\
\text { classrooms. } \\
\text { - Pretraining: students were trained for three } 45 \text {-min sessions on CWPT } \\
\text { procedures. } \\
\text { B. Classwide Peer Tutoring: CWPT consisted of } 30 \text { min peer-mediated } \\
\text { instruction } 3 \text { to } 4 \text { days a week. Activities done were reading of passages by } \\
\text { students, feedback from peers in oral reading, correction of errors and } \\
\text { comprehension questions from the peer tutor. } \\
\text { - Unstructured free-time groups: Students engaged in } 15 \text { to } 20 \text { min of social time } \\
\text { through activities selected by the teacher to promote social interactions. }\end{array}$ \\
\hline $\begin{array}{l}\text { 5. Kamps et } \\
\text { al. (1999) }\end{array}$ & $\begin{array}{l}\text { To investigate the use of a peer network } \\
\text { System including a cross-age tutoring } \\
\text { component to promote interaction time for } \\
\text { students with autism and their nondisabled } \\
\text { peers. }\end{array}$ & $\begin{array}{l}2 \text { students diagnosed } \\
\text { with ASD: } \\
\text { - Ann, 9-year-old. } \\
\text { - Kyle, 9-year-old. } \\
5 \text { typically } \\
\text { developing } \\
\text { students, 9-year- } \\
\text { old. } \\
6 \text { typically } \\
\text { developing } \\
\text { students, 6-year- } \\
\text { old. }\end{array}$ & $\begin{array}{l}\text { Multiple baseline design: A-B-A-B } \\
\text { A. Baseline 1: 20-min unstructured free time sessions with the first target student } \\
\text { (Ann) and five fourth grade peers. They were given no other instructions than } \\
\text { to play with a variety of materials. } \\
\text { B. Cross-age tutoring 1: tutors were involved in one week of training in tutoring } \\
\text { skills. After the week of training, } 6 \text { weeks of the tutoring program with first } \\
\text { graders as tutees was implemented. 20-min tutoring sessions: } 10 \text { minutes of } \\
\text { structured tutoring in a selected group of sight words and } 10 \text { mins of } \\
\text { unstructured free time. } \\
\text { A. Baseline 2: Same procedures of the Baseline } 1 \text { and the second target student } \\
\text { (Kyle). } \\
\text { B. Cross-age tutoring } 2 \text { : Brief retraining in tutoring skills for the participants. } \\
\text { Two new tutors were trained to implement tutoring. Two new first grade } \\
\text { students were added as tutees. }\end{array}$ \\
\hline $\begin{array}{l}\text { 6. Kamps et } \\
\text { al. (1989) }\end{array}$ & $\begin{array}{l}\text { To validate the effectiveness of a peer } \\
\text { tutoring program in facilitating the } \\
\text { acquisition of academic and social skills for } \\
\text { students with autism. }\end{array}$ & $\begin{array}{l}2 \text { students diagnosed } \\
\text { with ASD: } \\
\text { - Student 1, 9-year- } \\
\text { old. }\end{array}$ & $\begin{array}{l}\text { Multiple baseline design: A-B } \\
\text { A. Baseline: No changes were made in the classroom routines, to evaluate entry } \\
\text { level abilities for specific tasks. }\end{array}$ \\
\hline
\end{tabular}




\begin{tabular}{|c|c|c|c|}
\hline Reference & Purpose of the research & Participants & Intervention design / procedures \\
\hline & & $\begin{array}{l}\text { - Student 2, 11-year- } \\
\text { old. } \\
2 \text { typically } \\
\text { developing } \\
\text { students, 10-years- } \\
\text { old. }\end{array}$ & $\begin{array}{l}\text { - Tutor training: } 12 \text { 30-minutes sessions preparing the fifth-grade students to } \\
\text { become effective tutors. } \\
\text { B. Peer tutoring sessions: Tutors came to the special education classroom during } \\
\text { 30-minute three times } \\
\text { per week. Tutors provided one-to-one instruction for } 20 \text { minutes, three times per } \\
\text { week, on tasks based in } 3 \text { content areas: money skills, expressive language, and } \\
\text { oral reading/comprehension skills. The last } 10 \text { minutes of each session was } \\
\text { spent in free-play activities. }\end{array}$ \\
\hline $\begin{array}{l}\text { 7.Laushey } \\
\text { and Helfin } \\
(2000)\end{array}$ & $\begin{array}{l}\text { To determine if the peer tutoring training of } \\
\text { an entire class, including those with ASD, } \\
\text { would increase the generalization of social } \\
\text { skills across students. }\end{array}$ & $\begin{array}{l}\text { Two students } \\
\text { diagnosed with } \\
\text { ASD: } \\
\text { - John, 5-year-old. } \\
\text { - Pat: 5-year-old. } \\
\text { Other typically } \\
\text { developing } \\
\text { students, } 5 \text { /6-year- } \\
\text { old classmates. }\end{array}$ & $\begin{array}{l}\text { Multiple baseline design: A-B-A-B } \\
\text { A. Baseline 1: Target students were in their classes with nondisabled peers. } \\
\text { B. Active peer tutoring program: implemented during the free play center time } \\
\text { for all students. } \\
\text { A. Baseline } 2 \text { : The peer tutoring system was taken away and the children } \\
\text { returned to the passive proximity peer tutoring condition. } \\
\text { B. Active peer tutoring program was reinstated. } \\
\text { Pre-Training procedures: all students, including those with ASD, were trained to } \\
\text { stay with, play with and talk to a peer. }\end{array}$ \\
\hline $\begin{array}{l}\text { 8. McGee et } \\
\text { al. }(1992)\end{array}$ & $\begin{array}{l}\text { To analyze the efficacy of incidental teaching } \\
\text { as an intervention designed to promote } \\
\text { reciprocal peer interactions among children } \\
\text { with autism. }\end{array}$ & $\begin{array}{l}3 \text { children diagnosed } \\
\text { with ASD: } \\
\text { - Ian, } 3 \text {-year-old. } \\
\text { - Max: 4-year-old. } \\
\text { - Sam: } 5 \text {-year-old. } \\
5 \text { typically } \\
\text { developing } \\
\text { students, aged } 3 / 4 \\
\text { years old. }\end{array}$ & $\begin{array}{l}\text { Multiple baseline design: A-B-C-C } \\
\text { A. Baseline. } \\
\text { B. Peer training for incidental teaching: } \\
\text { 1). Peer tutor training: The teacher provided instruction to tutor about how to } \\
\text { conduct the teaching process. } \\
\text { 2). Peer tutoring intervention: The teacher presented the peer tutor with the } \\
\text { bucket of toys. Tutoring sessions between the peer tutor and the target child } \\
\text { took place. } \\
\text { C. Fading 1: The experimental teacher reduced her active involvement when the } \\
\text { tutor demonstrated the ability to conduct the incidental teaching process. } \\
\text { C. Fading 2: The teacher was no longer present in the room. }\end{array}$ \\
\hline $\begin{array}{l}\text { 9. Peturs- } \\
\text { dottir et al. } \\
(2007)\end{array}$ & $\begin{array}{l}\text { To explore the effects of scripted peer } \\
\text { tutoring in reading activities on social } \\
\text { interactions between a student with ASD } \\
\text { and his typically developing peer tutors. }\end{array}$ & $\begin{array}{l}1 \text { student diagnosed } \\
\text { with ASD: } \\
\text { - Max, 5-year-old. } \\
3 \text { typically } \\
\text { developing peers: }\end{array}$ & $\begin{array}{l}\text { Multiple baseline design: A-B-A, used to examine the effects of peer tutoring on } \\
\text { social interactions. } \\
\text { Multiple baseline design: A-C-B-C, used to examine the effects of programming } \\
\text { common stimuli in the peer tutoring reading activity and free-play time. } \\
\text { Programmed common stimuli: Toys with at least } 20 \text { pieces that could be put } \\
\text { together (marble run). }\end{array}$ \\
\hline
\end{tabular}




\begin{tabular}{|c|c|c|c|}
\hline Reference & Purpose of the research & Participants & Intervention design / procedures \\
\hline & & $\begin{array}{l}\text { - Rick, Joe and Bob, } \\
\text { 5-year-old. }\end{array}$ & $\begin{array}{l}\text { A. Baseline: 20-min free play periods following reading sessions. } \\
\text { B. Scripted peer tutoring: 20-min free play periods following peer tutoring } \\
\text { reading activities. } \\
\text { C. Scripted peer tutoring with common stimuli: Reading sessions in which play } \\
\text { related stimuli were programmed into the reading activities: the tutor praised } \\
\text { the reader after each line of text and asked him to take one toy item and place it } \\
\text { in an appropriate spot. }\end{array}$ \\
\hline $\begin{array}{l}\text { 10. Whita- } \\
\operatorname{ker}(2004)\end{array}$ & $\begin{array}{l}\text { To increase the frequency with which the } \\
\text { children with ASD initiated communication } \\
\text { and joint attention in the context of } \\
\text { interactive play sessions. }\end{array}$ & $\begin{array}{l}10 \text { students } \\
\text { diagnosed with } \\
\text { ASD, 6/7-year-old. } \\
10 \text { typically } \\
\text { developing peers, } \\
\text { 11-year-old. }\end{array}$ & $\begin{array}{l}\text { A. Training, coaching and support for tutors: The first four weeks, peer tutors } \\
\text { were asked if they can get their tutee to play with them. The adult's role was to } \\
\text { provide encouragement and specific intervention if necessary. } \\
\text { - Session of training: explanation, modelling and role play were provided for peer } \\
\text { tutors. } \\
\text { B. Regular play sessions: } 1 \text {. Opened with a simple constructional play; } 2 \text {. Pairs } \\
\text { had access to a range of toys and materials, selected on basis of children with } \\
\text { ASD preferences; C. Closing activity: parallel play in where all individuals were } \\
\text { performing the same action at the same time. }\end{array}$ \\
\hline
\end{tabular}

